\title{
Test Diagnosing Of Learning Activity
}

\author{
Roman Yavich, Ariel University, Israel \\ Alexander Gein, Ural Federal University, Russia \\ Alexandra Gerkerova, South Ukrainian Pedagogical University named after K. D. Ushinsky, Ukraine
}

\begin{abstract}
The technology of criteria-oriented testing enhanced by the reflexive components is suggested in this article. Tests made according to this technology are called academic activity tests. The student chooses or formulates not the answer to the problem but an action that is productive in his opinion. So, this type of tests helps not only check the accuracy of the achieved results, but traces the course of its getting. The main characteristics of such tests that make it possible to estimate it diagnostic potential are presented. Among these are variability of the test and the length of the minimum and maximum trajectory of the result achievement. The test of this kind with the discussion of its diagnostic possibilities is given as an example
\end{abstract}

Keywords: Criterion-Referenced Test; Diagnosis Logical Activity; Academic Activity Test

\section{INTRODUCTION}

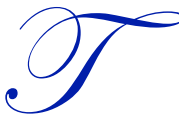

he use of test forms in the educational process is regarded as one of the major issues of pedagogical science and practice of the XXI century (Frederickson, Mislevy, \& Bejar, 1993, p. 404). The most important positive characteristics of test technologies are their objectivity, standardizing of the procedures, and better measurability of the outcomes due to the more flexible scale usage.

Up to the 60 -ies of the XX century the test methods used had two target areas:

- IQ tests;

- academic achievements tests.

Both cases are actually referring to the ranking of the testees in accordance with the statistically established norms. Hence this approach is called norm-referenced Keeves, 1988).

As for IQ-tests it should be noted that their contents did not bear much relation to the curriculum, so the correlation between the test success and academic achievements was, as a rule, although positive, rather low (less than 0.5).

Academic achievements tests are a form of knowledge and skills assessment acquired by students in the learning process. One of the major disadvantages of this test form is the inability to estimate how the answers were obtained by a testee. According to A. N. Mayorov (2000) "... the analysis of the problem solution methods and thinking operations that a pupil uses for solving the problems in most cases is hindered or impossible" (p. 38). This proves a sheer impossibility of such tests' usage in assessment of intellectual skills acquired at the time of learning.

In 1963, Robert Glaser suggested a criterion-referenced assessment as an alternative. This approach is characterized by orientation not on the statistical norm but on a given objective substantive standard - standard execution - with which the results of each test are compared. The consequence is that in norm-referenced tests a task is not considered valid if it is fulfilled by all the testees; in criterion-referenced tests, such a task, it is not only appropriate, but also serves as a basis for concluding about the sufficient level of learning material retention by all the students.

At the initial stage the criterion-referenced tests were aimed at solving the same estimation problem of students' academic achievements. But in the mid-80s as a result of this technology study, it became clear that this approach can 
be effectively used for the diagnosis of students' operational and logical activity in the course of tests' carrying out. In other words, it is a diagnosis of students' mental activity (Starichenko, Egorov, \& Yavich, 2013, p. 23).

\section{CRITERION-REFERENCED TEST}

Here is an example of criterion-referenced test made up for the diagnosis of such a mental action as a definition of the classification principle.

Four tasks are given: three are related to the problems of one kind, and one is related to another. They are denoted as A, B, C, D.

A. A Farmer planes on plowing 150 acres daily. However, due to the good weather, he was able to plow up to $25 \%$ daily, and therefore, all the work was completed one day earlier than expected. How long did it take him to plough the field?

B. The computer-controlled machine manufactures 25 details per hour. After optimizing of the program, it started to manufacture 3 more details per hour. It made it possible to fulfill an order three hours faster. What was the order volume?

C. It takes the train 1.2 hours to pass the distance between the two stations. Because of track repair the train decreased its speed by $20 \%$ and went the distance in 1.5 hours. Find the initial speed of the train.

D. Two workers were set to make 8,840 details. Because of the difference in abilities the productivity of the first worker is $35 \%$ higher than of the second one. After four days' work, the first worker was transferred to another job. It took the second worker one more day to fulfill the order. How many details were produced by each worker?

The essential feature of this test is that while performing it, a student does not solve the problems but compares and combines the tasks on the basis of generality of the relation types between values (task D is an odd one out). The generality of the plot (do some work - tasks A, B, D), the similarity of the individual parts (the relationship between the values of the quantities given in the form of percentages - tasks A, B, D) are not sufficient grounds for concluding that the tasks are related to one another and are the same type. Students' choice of classification criterion makes it possible to estimate their analytical mental activity.

However, the solution of the problem doesn't reduce to the classification procedure, it unfolds as a holistic sequence of mental operations. In our opinion, for the effective diagnosis of such activities, the technology of academic activity tests, which has been developed since the early $90 \mathrm{~s}$ of the last century should be used. The extensive study of this technology was held by Senognoeva (2013). However, this technology should be completed with some tools of reflexive nature for the diagnosis of the academic activity, not just its results.

The gist of the academic activity test technology is that unlike the standard test, where the student should choose one of the answers or just write down the result he got (after that the teacher can only form a hypothesis why the student got such a result), the academic activity test suggests choosing one operation of several alternatives at every step. Every time an operation is estimated to be a productive one (i.e. leading to the problem solution) or a dead-end. As a result of these steps a solution is arrived at, i.e. the student always has a solved problem as a result. This is very important from the psychological point of view.

The principal characteristic of an academic activity test is that there are always different ways of solution approaches to one and the same problem. These ways of solution may be of different length depending on the choice of the theory that the student will use for the problem solution, on the level of his knowledge and skills etc. so the academic activity tests could be regarded as one of the paradigms of the adaptive training systems (Senognoeva, 2013); however, the adaptation is not effected by the level of difficulty, but estimates whether the mental action is correct.

It is important that the choice of action at one or another stage of the test is the projection of an appropriate mental action. We can note how well the student can select the information and separate essential from the nonessential, convert it to sign-symbolic form and operate with it, his mental actions being expanded or contracted. 
The fundamental development of the academic tests technology in our work is the inclusion of items allowing the students to carry out reflection of their actions. The fact is that the competence formation cannot be fully realized if the student doesn't self-reflect his activity as universally valid, i.e. as applicable to the different starting situations.

Note that this «reflexive» addition to the test tasks brings them to better correspondence with the psychologicalpedagogical concept of "academic activity". By Davydov's (1982) interpretation, an academic activity is the one of a subject in mastering of the methodology of learning actions and self-development in the process of training problems solution on the base of external monitoring and estimation, turning into self-control and self-estimation (Keller, Yavich, Domoshnitsky, 2014, p. 133).

In the light of the foregoing we will mention that the solution of training problems is not a goal but a means of the goals' achievement, so the role of a training problem is that the students master the common ways of working with the material and transfer them to the solution of the problems where these methods are applicable. In the problem that represents a complex system of information about some phenomenon, object, process, only some facts are given. The rest information is unknown and should be found out on the basis of the other data, through the establishment of the links between them, transformations fulfillment etc.

So, the method of a problem solution is any procedure that leads to the task solution. In other words, the way of a problem solution correlates the subjective characteristics of the person and problem solver that determine both the selection of the operations sequence and the general strategy of the solution. The solution of the problem in different ways provides more opportunities for the development of a student. While solving the problem by the only one method the goal of the student is to find the correct answer; using several ways of the problem solution he faces the choice of the shortest and most efficient one. It requires the actualization of theoretical knowledge and many known techniques and methods or the creation of the new methods and techniques applicable to the situation in the problem. Herewith the students accumulate experience of knowledge application that contributes to the development of logical search techniques and, in turn, develops their research abilities.

In the process of looking for solution, a student can or often has to try different strategies and methods. The algorithm of problem solution is a chain of interrelated, serendipitously chosen statements. Such chain building is one of the main academic skills, the basis of the scientific activity. The final step of the solution is the choice of the strategy, realized by the definite way of proceeding. The pattern of a training problem solution together with the tentative one includes other parts of the mode of action, primarily controlling and executive. Unimpaired operation of an academic activity assumes well-formedness of all the parts of the mode of action.

We can single out the following parts of the mode of action:

- Finding the backgrounds for the academic activity success (the student should realize what he knows and is able to do and the way it correlates with the problem)

- The choice of the general strategy

- Determination of the missing information

- Search for the missing information

- Search for confirmatory evidence for the chosen way of solution propriety

- Decomposition of the main problem into the sub-problems

- Solution of the sub-problems

- Choice of the most effective algorithm of actions

- Execution of the operations appropriate in this particular case.

Therefore, the problem solution goes through the realization of some academic actions sequence. The student should have an idea about the academic activity components, the ways of these actions carrying out and their sequence.

Knowledgeable fulfillment of an academic task at all stages and timely correction of the errors are achieved by means of step-by-step checking. A student must not only identify the difficulty that arose in the course of solving the problem, but also realize to what step of solution it refers. For academic activities tests it is very important that the step-by-step Copyright by author(s); $\underline{\text { CC-BY }}$ 
checking can refer both to the carried out and to planned actions. In turn, it makes it possible to analyze the work on the task before its actual execution, to anticipate the results. The students, who mastered such form of control, do not start the task execution the moment they get it. At first they make an action plan, compare and estimate the ways of solution, choose the most appropriate ones, think over the possible consequences of their usage and only after that set out on the task. If a need arises, they can return to every step of the task fulfillment, check the carried out actions, find the false ones and correct them. Academic activity becomes autonomous and self-regulatory. A student, having mastered such activities is able to foresee the occurrence of possible errors and difficulties and to schedule timely measures to prevent them.

As previously stated, the task in the academic activity test is a set of different ways, each leading to the solution of the problem, but these ways are different in composition of the actions, suggested for their fulfillment.

The first step along the way of the test task is the elicitation of all the concepts used in the stated problem. The direction of the next step is chosen by the student depending on how deeply he/she has understood the contents of each of the selected concepts and how he understands the interrelations of the concepts. These links may already be known or can be easily established by the student due to their knowledge and skills or may turn out to be an auxiliary task that will require the straight-line diagram of this relation. Similarly, the second step is carried out, etc. Therefore, each way has its own length. The shortest way corresponds to be degree of student's being familiar with the material at the highest level of convolution. If on a particular step a subtask occurs that requires a separate decision, it is carried out after the same pattern. In other words, the problems in the tests have the structure of a fractal type.

The ways of the problem solution, the level of its convolution to a great extent depend not only on the problem itself, but on the facts and methods learnt by the student by the time of the test.

An additional advantage of testing is possible using interactive computer technologies. The implementation of academic activity tests without computers is next to impossible. Firstly, the goal of the test fulfillment is not the obtainment by the student of a single answer, but finding a rather long way where the subsequent step depends on the previous one and the analysis of every selected answer should be presented to the student so that he did not know the analysis of the other answers. Secondly, for a full analysis it is necessary to have a complete record of the test task fulfillment for each student. Third, due to the considerable volume of the fixed information about the test fulfillment it is necessary to have the set of tools information processing implemented in the form of computer software.

We will cite the example of the training test. For easy perception we will consider a fairly simple task of school geometry (in particular, it means that students do not know the trigonometric functions of the angle and cannot use them to solve this problem).

The students should solve a text problem. The testing is computer-based; the presentation of the test tasks, the variants of answers and the analysis of the students' choice are implemented to interactively. To describe the order of the assignments presentation with all the alternatives and analysis we use the following techniques: the tasks are presented to the students consistently (they are numbered from 1 to 24 - this is the total number of tasks in this test), the answers to every task are marked by the letters of the alphabet. After choosing the answer the student is given an analysis and the instructions on what to do next (the analysis is marked by a combination of the letter D and the analysis serial number). In addition, we provide diagnostic comments about the nature and the level of learning actions development. Students do not see those comments, but the teacher may obtain them; access to them is protected by a teacher's individual password.

\section{THE PROBLEM}

The diagonal $A C$ of the parallelogram $A B C D$ makes the angle $30^{\circ}$ with the side $A B$. Solve the area of the parallelogram if $A B=6 \mathrm{~cm}$ and $A C=8 \mathrm{~cm}$. 


\section{Instruction}

To solve the problem, you should execute the steps in sequence; starting with p. 1 and each time choose one of the suggested answers.

P. 1. For the solution of the problem, the following information will be useful:

\begin{tabular}{l|l|l}
\hline A. The definition of a parallelogram. & $\rightarrow$ & D1 \\
\hline B. The properties of a parallelogram. & $\rightarrow$ & D2 \\
\hline C. The formula for solving the area of a triangle. & $\rightarrow$ & D3 \\
\hline D. The formula for solving the area of a parallelogram. & $\rightarrow$ & D4 \\
\hline E.Other information. & D5 \\
\hline
\end{tabular}

D1. Remember the definition and think whether it can help you to define other parameters of the parallelogram. Consider another variant. Return to p. 1

D2. This way is possible. Go to p. 2.

D3. This way is possible. Go to p. 11 .

D4. This way is possible. Go to p. 12 .

D5. Write down your own version in the notebook and discuss with the teacher wants to do next.

\section{Diagnostic Comment}

If a student chooses $\mathrm{A}$ then it is indicated that in this task they will be guided on only outward sign: on the fact that the parallelogram is mentioned in the problem. Here we can diagnose insufficient formation of such a universal mental action as the ability to identify and formulate essential information for this problem solution.

If the student chooses B then it is likely that the overall plan of solution is still absent, but they understand that the definition of a parallelogram is unlikely to be helpful in itself, and assumes that there are properties that allow to them to solve the problem.

If the student chooses $\mathrm{C}$ then, although such choice may lead to the problem solution, it is likely that this choice is made because the triangle $\mathrm{ABC}$ is defined clearly, i.e. all its characteristics, including the area, can be calculated one way or another. It is pretty typical but in general it is a counterproductive strategy of trials and errors, when instead of trying to make a general plan of the goal achievement, a student takes steps at random simply because they are feasible.

The choice of D is strategically correct since as it is known making a plan for a problem solution should be started with the analysis of what you want to solve (Chelyshkova, 2001, p. 165). In this case, the area of the parallelogram is being solved so the choice of the answer is quite natural. However, this does not mean that the student already has a full plan for the problem solution, the final conclusion can be made after fulfillment of the subsequent test tasks.

The choice of E will not lead to the solution. In this case the diagnosis is the same as that shown in D1, of course, with the adapted commentary why that choice is untoward.

P. 2. What property of the parallelogram is useful for the problem solution in your opinion?

\begin{tabular}{l|l|l}
\hline A. The opposite sides of a parallelogram are equal. & $\rightarrow$ & D6 \\
\hline B. A diagonal of a parallelogram divides it into two equal triangles. & $\rightarrow$ & D7 \\
\hline C. The diagonals of a parallelogram bisect at the cross point. & $\rightarrow$ & D6 \\
\hline D. Other property. & $\rightarrow$ & D8 \\
\hline
\end{tabular}


D6. Think whether this property will help to define some other parameters of the parallelogram. Consider another variant. Return to p. 2.

D7. This is useful information. Go to p. 3.

D8. Write down your own version in the notebook and discuss with the teacher what to do next.

\section{Diagnostic Comment}

If the student chooses the $\mathrm{B}$, this enhances the likelihood of a hypothesis that he already has a plan of the problem solution. If he chooses $\mathrm{A}$ or $\mathrm{C}$ then it means that he has no plan. If he chooses $\mathrm{D}$, then either he gives a formulation which is equivalent to B but he does not understand it (this shows the problems of his logical thinking), or his formulation is equivalent to the choice of $\mathrm{A}$ or $\mathrm{C}$ with the corresponding diagnostics.

P. 3. To solve the area of a triangle it is enough to know:

\begin{tabular}{l|l|l}
\hline A. The lengths of all sides of the triangle. & $\rightarrow$ & D9 \\
\hline B. The length of the side and the height dropped on this side. & $\rightarrow$ & D10 \\
\hline C. The lengths of the two sides of the triangle and the angle between them. & $\rightarrow$ & D9 \\
\hline D. Other numerical information on the triangle. & D11 \\
\hline
\end{tabular}

D9. It's correct, but your knowledge for needed calculations is not enough. Consider another variant. Return to p. 3.

D10. It's correct. Go to p. 4.

D11. Write down your own version in the notebook and discuss with the teacher what to do next.

\section{Diagnostic Comment}

As in p. 2, the choice of the correct alternative enhances the likelihood of a hypothesis that the student already has a full plan the problem solution; the choice of the incorrect alternative indicates faulty interpretation of his knowledge for the problem solution.

P. 4. To solve the area of the triangle $\mathrm{ABC}$ you choose:

\begin{tabular}{l|l|l}
\hline A. The side $A B$ and the height dropped on it. & $\rightarrow$ & D12 \\
\hline B. The side $B C$ and the height dropped on it. & $\rightarrow$ & D13 \\
\hline C. The side $A C$ and the height dropped on it. & $\rightarrow$ & D14 \\
\hline
\end{tabular}

D12. This way is possible. Go to p. 5 .

D13. This way is possible but your knowledge is not enough for the necessary calculations. Consider another variant. Return to p. 4.

D14. This way is possible. Go to p. 6 .

\section{Diagnostic Comment}

Here is no variant with the appeal to the teacher because the choice of the alternatives is exhausted. The actions suggested in A and C lead to the problem solution, since we can say with confidence that student has a developed plan for the problem solution. For the variant D13 the comment is the same as for D9. 
P.5. To solve the height $\mathrm{CH}$ dropped from the vertex $\mathrm{C}$ on the side $\mathrm{AB}$ you will consider:

\begin{tabular}{l|l|l}
\hline A. The right triangle $A C H$. & $\rightarrow$ & D15 \\
\hline B. The right triangle $B C H$. & $\rightarrow$ & D16 \\
\hline C. Another geometric figure. & $\rightarrow$ & D17 \\
\hline
\end{tabular}

P. 6. To solve the height $B H$ dropped from the vertex $B$ on the side $A C$ you will consider

\begin{tabular}{l|l|l}
\hline A. The right triangle $A B H$. & $\rightarrow$ & D15 \\
\hline B. The right triangle $B C H$. & $\rightarrow$ & D16 \\
\hline C. Another geometric figure. & $\rightarrow$ & D17 \\
\hline
\end{tabular}

D15. It's correct. Go to p. 7.

D16. Note that in this case you will not be able to use the information on the angular size $B A C$. Consider another variant. Go to the same point.

D17. Write down your own version in a notebook and discuss with the teacher the choice of next point.

\section{Diagnostic Comment}

If $\mathrm{A}$ or $\mathrm{B}$ have been chosen, then purely technical part of the problem solution is remained. The choice of $\mathrm{C}$ means, that actually all the previous steps have been taken at random.

\section{P. 7. The cathetus of the right triangle lying opposite to the angle of $30^{\circ}$ is equal}

\begin{tabular}{|c|c|c|}
\hline A. The half of the hypotenuse of the triangle. & $\rightarrow$ & D18 \\
\hline B. Another cathetus. & $\rightarrow$ & D19 \\
\hline C. The half of the another cathetus. & $\rightarrow$ & D19 \\
\hline D. Other value, different from the mentioned in A $-\mathrm{C}$. & $\rightarrow$ & D20 \\
\hline
\end{tabular}

D18. It's correct. Go to p. 8 .

D19. You don't know the property of the cathetus lying opposite to the angle of $30^{\circ}$. Consider another variant. Return to p. 7.

D20. Write down your own version in a notebook and discuss with the teacher the choice of next point.

\section{Diagnostic Comment}

It is testing of the students' actual knowledge. If a student chooses D, then either he gives a formulation which is equivalent to $\mathrm{A}$, but he does not understand it (this indicates the problems in his logical thinking), or his formulation is equivalent to $\mathrm{B}$ or $\mathrm{C}$ with the corresponding diagnostics.

P. 8. The area of the triangle is equal to

\begin{tabular}{l|l|l|}
\hline A. The product of the triangle height by the base. & $\rightarrow$ & D21 \\
\hline B. The half the product of the triangle height by the base. & $\rightarrow$ & D22 \\
\hline C. The value calculated differently than described in A or B. & $\rightarrow$ & D23 \\
\hline
\end{tabular}

D21. You don't know the triangle area formula. Consider another variant of answer. Return to p. 8.

D22. It is correct. Go to p. 9.

D23. Write down your own version in a notebook and discuss with the teacher the choice of next point. 


\section{Diagnostic Comment}

Here as in the previous task, the student's actual knowledge is examined.

1. Calculate the area of the triangle $A B C$ and enter the answer:

If the answer is correct then transfer to p. 10, otherwise return to p. 9.

2. Calculate the area of the parallelogram $A B C D$ and enter the answer:

If the answer is correct then the fulfillment of the test task is completed, otherwise return to p. 10.

P. 9. How is the area of the triangle $A B C$ and area of a parallelogram $A B C D$ interrelated?

\begin{tabular}{l|l|l}
\hline A. The area of the triangle is equal to half the area of a parallelogram. & $\rightarrow$ & D24 \\
\hline $\begin{array}{l}\text { B. The area of the triangle is a quarter of the area of a parallelogram. } \\
\begin{array}{l}\text { C. The relation between these values depends on the shape of a parallelogram - whether } \\
\text { it is a rectangle, rhombus, etc. }\end{array}\end{array}$ & $\rightarrow$ & D25 \\
\hline
\end{tabular}

D24. It's correct. Go to p. 3 .

D25. You do not know the correct interrelation between the areas of the triangle and the parallelogram. Consider another variant. Go to p. 11.

Diagnostic Comment

The choice of A or B means that the student has an overall plan of the problem solution, but in the case B it would lead to an erroneous result. The choice of $\mathrm{p}$. C confirms the hypothesis about the choice of the answer in $\mathrm{p}$. 1 exclusively oriented on the source data rather than on that is necessary to solve in the problem.

P. 10. To solve the area of a parallelogram it is sufficient to know

\begin{tabular}{l|l|l}
\hline A. The lengths of all sides of the parallelogram. & $\rightarrow$ & D26 \\
\hline B. The length of one of the sides and the height dropped on this side & $\rightarrow$ & D27 \\
\hline C. The lengths of two adjacent sides of the parallelogram and the angle between them. & $\rightarrow$ & D28 \\
\hline D. Other numerical information on the parallelogram. & $\rightarrow$ & D29 \\
\hline
\end{tabular}

D26. It isn't correct; remember that the parallelogram is not a rigid figure. Consider another variant. Go to p. 12.

D27. It's correct. Go to p. 13.

D28. It's correct, but your knowledge is not enough for the necessary calculations. Consider another variant of answer. Go to p. 12 .

D29. Write down your own version in a notebook and discuss with the teacher the choice of next point.

\section{Diagnostic Comment}

The choice of A means a random walk in the search of a solution. The choice of the correct alternative B enhances the likelihood of a hypothesis that the student already has a full plan of the problem solution. The choice of the alternative, $\mathrm{C}$, indicates misestimating of the student's knowledge necessary for this problem solution. 
P. 11. To solve the area of the parallelogram you choose:

\begin{tabular}{l|l|l}
\hline A. The side $A B$ and the height dropped on it. & $\rightarrow$ & D30 \\
\hline B. The side $B C$ and the height dropped on it. & $\rightarrow$ & D31 \\
\hline C. The side $C D$ and the height dropped on it. & $\rightarrow$ & D32 \\
\hline D. The side $A D$ and the height dropped on it. & $\rightarrow$ & D31 \\
\hline
\end{tabular}

D30. This way is possible. Go to p. 14 .

D31. Note that in this case you will not be able to use the information on the value of the angle $B A C$. Consider another variant. Go to p. 13.

D32. This way is possible. Go to p. 17.

\section{Diagnostic Comment}

The choice of B or D means that the student still has no any clear plan for the problem solution, it is likely that he is moving on the trajectory that is paved by this training test, without clear awareness of the performed actions goal. If a student chooses $\mathrm{A}$ or $\mathrm{C}$, then the degree of the planned awareness will be shown in the process of the subsequent tasks fulfillment.

P. 12. To solve the height $C H$ dropped from the vertex $C$ on the side $A B$ you will consider

\begin{tabular}{l|l|l}
\hline A. The right triangle $A C H$. & $\rightarrow$ & D33 \\
\hline B. The right triangle $B C H$. & $\rightarrow$ & D16 \\
\hline C. Another geometric figure. & $\rightarrow$ & D17 \\
\hline
\end{tabular}

D33. It's correct. Go to p. 15 .

\section{Diagnostic Comment}

This paragraph coincides with p. 5, so the comments are the same. We have to use the diagnostics D33 (instead of D15), since the subsequent trajectory will be different than after p. 5.

P. 13. The cathetus of a right triangle lying opposite to the angle of $30^{\circ}$ is equal to

\begin{tabular}{l|l|l}
\hline A. The half of the hypotenuse of the triangle. & $\rightarrow$ & D34 \\
\hline B. Another cathetus. & $\rightarrow$ & D19 \\
\hline C. The half of the another cathetus. & $\rightarrow$ & D19 \\
\hline D. Other value, different from the mentioned in A - C. & $\rightarrow$ & D20 \\
\hline
\end{tabular}

D34. It's correct. Go to p. 10.

\section{Diagnostic Comment}

This paragraph coincides with p. 7, so the comments are the same. We have to use the diagnostics D34 (instead of D18), since the subsequent trajectory will be different than after p. 7.

P. 14. To solve the length of the side CD it is necessary to use

\begin{tabular}{l|l|l}
\hline A. the properties of the opposite sides of the parallelogram. & $\rightarrow$ & D35 \\
\hline B. the properties of the triangles into which a diagonal divides a parallelogram. & $\rightarrow$ & D35 \\
\hline C. the properties of triangles into which the diagonals divide the parallelogram. & $\rightarrow$ & D35 \\
\hline
\end{tabular}

D35. This way is possible. Go to p. 17. 


\section{Diagnostic Comment}

In all cases the conclusion of the equality of the sides $A B$ and $C D$ is made, but in fact, this way is the elongation of the way $13-14-15-10$.

P. 14. To solve the height $A H$ dropped from the vertex $A$ on the side $C D$ you will consider

\begin{tabular}{l|l|l}
\hline A. The right triangle $A C H$. & $\rightarrow$ & D36 \\
\hline B. The right triangle $A D H$. & $\rightarrow$ & D16 \\
\hline C. Another geometric figure. & $\rightarrow$ & D17 \\
\hline
\end{tabular}

D36. It's correct. Go to p. 15 .

Diagnostic Comment

Essentially this point coincides with p. 14, so the comments are the same.

\section{RESULTS}

To visualize the set of trajectories of the problem solutions, it is convenient to represent them by a directed graph. The vertices are the paragraphs of test task, and the ribs are the transitions from one paragraph to another. For the considered test task the corresponding graph is shown in Figure 1.

Figure 1. The structure of the academic activities test

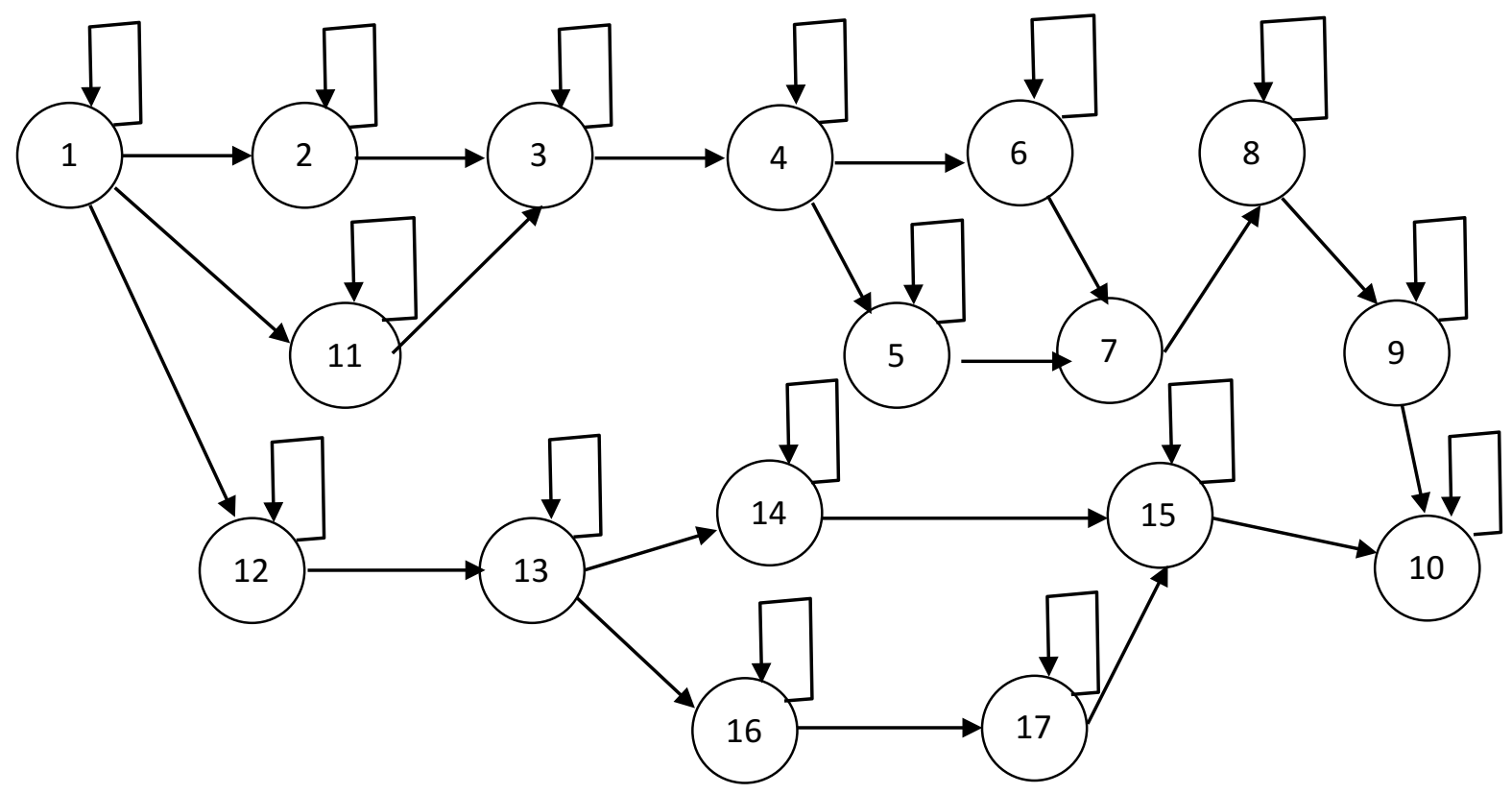

One of the important objective characteristics of the academic activity test is the number of possible ways (without loops), leading from the initial vertex to the end vertex. It is naturally called the variability of the test (Gorbachova (1988) called it the width of the test) since it shows a variety of possible approaches to the problem solution provided in the test. Such variety makes it possible to display the individual characteristics of students' thinking as well as the peculiarities of practical usage of their knowledge and skills. Our experiments with academic activity tests show that the good test's range of variability should be no smaller than three. However, if variability is too big, it is undesirable and the student's test results are difficult to compare. In our opinion, the upper limit of variability should not exceed eight. The variability of the reviewed test is equal to six, thus, it is within the boundaries required by this parameter. 
The second objective characteristic has a complex character and is described by three values: the length of the longest way from the initial vertex to the end (without loops), the length of the shortest way between the vertices and the ratio of these numbers. The meaning of this characteristic is clear: it allows us to estimate the extent to which the student is able to choose the best problem solution. It is clear that the ratio should be larger than 1 otherwise the test does not diagnose this student's ability. For our test this ratio is 1.8; in our view this is an acceptable value for the diagnosis. But the establishment of the optimal limits for this parameter requires further research.

With regard to the length of the longest way between the start and end vertices the choice of the optimal range for this value depends on of students' age and the level of their intelligence development.

The matter already mentioned, the actual length of the test fulfillment depends on how many times the student had to go back to the same paragraph until he found a productive continuation. The ratio of actual length to the nominal length shows to what extent the student solved the problem consciously, i.e. the level of appropriate mental actions development. The maximum possible value of the actual length can be determined a priori for each way from the initial vertex to the end vertex. The ratio of this value to the nominal length of the route is natural to call the diagnostic ability of test task. For our task this characteristic for various routes averaged 2.3. Our experiments show that good separation of students according to these diagnostics occurs if this characteristic lies in the range from 1.8 to 3 .

\section{DISCUSSION AND CONCLUSIONS}

Theoretical underpinning and experimental proof let us make the following conclusions:

1. The suggested technology (unlike tests that are designed only to get an answer) makes it possible to follow the path of the student's mental actions in the process of the problem solution. It provides an opportunity to state the necessary pedagogical techniques to improve the learning efficiency.

2. For traditional ways of testing such important characteristics as reliability, validity etc., that help to differentiate the students according to their academic achievements are set a posteriori, that is based on the results of the tests of the control group. The suggested technology allows to estimate these characteristics a priori

3. The suggested technology besides the diagnostic has also a learning effect. While test undergoing the student has to analyse his strategy for the problem solution, choosing the most productive way.

Currently the suggested technology has worked out to greater extent for Mathematics and Science. Its usage for Economics, Social science and Humanities requires an additional research.

\section{ACKNOWLEDGEMENTS}

Supported through the Competitiveness Project (Agreement between the Ministry of Education and Science of the Russian Federation and the Ural Federal University No. 02.A03.21.0006, 27.08.2013).

\section{AUTHOR BIOGRAPHIES}

Dr. Roman Yavich specializes in informatics. He deals with the use of Internet technologies in the educational process. Dr. Yavich is the author of the technical support concept in the Internet MathOlympiad organization. He is a member of the Steering Committee of the Israel Mathematical Olympiad (on behalf of the Ministry of Education). Areas of interest include: Distance Education, ELearning, Networked, Education, Pedagogy. Academic administration: Referent of E-learning-Professional consultation for all the Faculties and Departments, in the field of E-learning, developing computer based training software and computers use in teaching process improvement. E-mail: (romany@ariel.ac.il)

Dr. Gerkerova Alexandera M. - Lecturer, Department of Germanic Philology and Foreign Languages teaching methodology. South Ukrainian National Pedagogical University named after K.D., Ushynsky, Odessa, Ukraine Email: gerkerova@ukr.net 
Dr. Alexander Gein Ph.D. in Education, Ph.D. in Physics and Mathematics, Professor in the Faculty of Mathematics and Computer Science at Ural Federal University (Russia). He has authored 100+ journal publications on mathematics and pedagogy and 10+ textbooks on computer science for secondary schools. E-mail: a.g.geyn@urfu.ru

\section{REFERENCES}

Chelyshkova M. B. (2001). Adaptive testing in education (theory, methodology, technology) Moscow: Research center of the quality problems of professional training, 165 .

Davydov V. V. (1982). The content and structure of schoolchildren's educational activity - Moscow: Pedagogy, 10-21.

Frederiksen N., Mislevy R.J., \& Bejar I. J. (Eds). (1993). Test theory for a new generation of tests. Lawrence Erlbaum Ass. Publ., Hillsdale, N-J., 404.

Glaser, R. (1963). Instructional technology and the measurement of learningoutcomes: Some questions. American Psychologist, $18,519-521$.

Gorbachova, E. I. (1988). The criterion-referenced testing in the diagnosis of mental development of schoolchildren. Voprosy psikhologii, 2, $51-57$.

Keeves J.P. (Ed.). (1988). Educational Research, Metodology and Measurement: An International Handbook. - Oxford: Pergamon press.

Keller, N., Yavich, R., \& Domoshnitsky, A. (2014). Mathematical debates as a form of student peer education-an additional link in the educational process. Far East Journal of Mathematical Education, 12(2), 133.

Majorov, A. N. (2000). Theory and practice of tests making for the education system (How to choose, make and use the tests for educational purposes). Moscow: Popular education, 38.

Senognoeva N. A. (2013). Tests of academic activity: innovative educational technology. Principles of formation and effectiveness of academic activity tests usage. Lambert Academic Publishing. Saarbrücken, Germany.

Starichenko, B. E., Egorov, A. N., \& Yavich, R. (2013). Features of application of classroom response system at the lectures in Russia and Israel. International Journal of Higher Education, 2(3), 23. 